\title{
Investigating the improvement of Degradation Resistant with the Addition of SDBS Anionic Surfactant to PEO polymer
}

\author{
Sarmad K. Fakhruddin ${ }^{1,2}$ *, Hayder A. Abdulbari ${ }^{1,3}$, Ahmad Z. Sulaiman ${ }^{l}$ and Hind A. \\ Rafeeq ${ }^{2}$, \\ ${ }^{1}$ Faculty of Chemical and Natural Resources Engineering, Universiti Malaysia Pahang, Gambang \\ 26300, Kuantan, Pahang, Malaysia. \\ ${ }^{2}$ Petroleum engineering department, Engineering College, University Kirkuk, 36001 Kirkuk, Kirkuk, \\ Iraq. \\ ${ }^{3}$ Centre of Excellence for Advanced Research in Fluid Flow, Universiti Malaysia Pahang, Gambang \\ 26300, Kuantan, Pahang, Malaysia
}

\begin{abstract}
Polyethylene Oxide (PEO) is one of the most common drag reduction agents. However, its ability to reduce drag in turbulent flow decreases with the time due to the degradation of its molecules. Thus, the enhancement of its degradation resistance by the addition of Anionic surfactant is presented. The Polyethylene oxide (PEO) was used in different concentration ranges from 10 to $60 \mathrm{ppm}$ and with addition of sodium dodecyl benzene sulfonate (SDBS) as the anionic surfactant with five concentrations between 100 to $500 \mathrm{ppm}$. The degradation measurements were done using rotating disk apparatus (RDA). The RDA results have shown a considerable improvement in the degradation resistance of PEO with the addition of surfactant to the polymer solution. The interaction between the polymer and the surfactant results in transferring the polymer chain from coil to straight-like body. Thus, enhance the polymer degradation resistant ability.
\end{abstract}

\section{Introduction}

The drag reduction was discovered by Toms in 1948, which is probably the effect produced by polymer addiction in fluids which has attracted most of the attention, due to its relevance for several applications. While performing experiments on the degradation of polymers, Toms observed that the addition of few parts per million of long chain polymers in turbulent flow produces a dramatic reduction of the friction drag [1]. Lately, several researches rapidly developed in the field of polymers additive to reduce the drag.[2] Drag-reducing polymers solutions are usually shear thinning, viscoelastic and either strain thinning or strain thickening, and to varying degrees each of these characteristics is thought to influence the level of drag reduction [3]. Polymeric DRA is proved to reduce transverse flow gradient, and then effectively creating laminar flow in the pipe. This phenomenon is usually occurring

* Corresponding author: sarmadfakhrulddin@gmail.com 
close to the pipe wall where axial flow velocity profile has a very steep gradient in which significant pressure losses happen [4]. Janosi et al, 2004 investigated in dam break flows, where a finite volume of fluid is released from a compartment into a long, rectangular channel. The result reported drag reduction occurs in the present of a minute amount of PEO (polyethylene oxide in different time range). The result proved that flow is much faster in the present of PEO which one of the polymer chain which has high molecular weight [5].

Polymer is very effective DRA but it is easily degraded in short time of the additive period. The major problems exist in the polymer degradation is due to mechanical force in solvents flowing at high velocity. The shear force at the wall of the flow channel will degrade the polymer mechanically [6].This mechanical stress will break the linkage of polymer hence reduces its efficiency towards the solvent [7]. To solve these major problems, there are three ways to enhance polymer stability and mechanical strength. The first one is polymer grafting to improve its molecular weight [8]. Grafted polymer is the way to enhance the molecular weight of the polymer by increasing bonding between polymer and increase polymer molecular weight and increase withholding from shear force in flow [9]. However, this method is too costly and needs précises operation conduction which makes it less attractive. The second method to enhance the polymer is by reversible intermolecular association. The objective is to improve mechanical stability by increasing molecular weight. There is the also cross-linking method that joining the polymer by another polymer to become like- ladder structures, but this method is not really efficient on shear degradation. The third method which is the most effective method is by the molecular interaction between the polymer and the surfactant $[10,11]$.

The field of polymers-surfactant application still limited with counted numbers of researches. Nonetheless, the result of the studies, that has been introduced the implementation of drag reduction using the polymer-surfactant complex, showed the improvement in the system in term of sustainability. Matras et al have studied poly (ethylene oxide) (PEO)cetyltrimethyl ammonium bromide (CTAB) complex formation and its effect on drag reduction. It was found that $\mathrm{PEO}-\mathrm{CTAB}$ aggregates reduced drag much more efficiently than these substances alone. Structure degradation appeared later and the drag reduction existed longer [12]. Moreover, damaged structure could be partly rebuilt. Kim et al studied the efficacy of complex additive that has same charge ionic polymer is poly acrylic acid (PAA) and anionic surfactant is sodium dodecyl sulfate (SDS) complex system regarding polymersurfactant interaction in a rotating disk apparatus on turbulent drag reduction (DR). They were concluded that, The confirmation of PAA-SDS mixture was to be more effective than that of pure PAA on drag reduction [13].

In addition, Mohsenipour and Pal, investigated the effect of formation complex microstructure of a mixed non-ionic polymer PEO and anionic surfactant SDS, in a pipeline flow loop on drag confinement. They found that the mixture of polymer-surfactant as a drag reducer shows a considerable synergistic effect on drag reduction (lower friction factors) as compared with system has polymer or surfactant alone. The effective of bonding polymersurfactant system is a stronger appearance at low additives concentrations ratio of polymer and surfactant. Although an improvement in resistance to shear degradation is observed when either SDS or OTAC is added to PEO, the effect is more prominent in the case of anionic surfactant SDS due to the size of micelles.[14] As the addition of anionic surfactants to nonionic polymer chains PEO causes stretching of molecules chains, it can be concluded that the expansion of polymer chains makes them less susceptible to mechanical degradation. As the improvement take steps forwards, future windows in saving the energy are opened.

This research presents the enhancement of PEO degradation resistance ability at very low concentration of PEO with the addition of SDBS anionic surfactant. The addition of SDBS to the polymer was investigated in term of the degradation resistance even at very low polymer concentrations and how can delay the mechanical degradation of polymer with time. 


\section{METHODOLOGY}

\subsection{Materials and Solution preparation}

Sodium dodecyl benzene sulfonate (SBDS) was used as anionic surfactant with molecular weight of $348.5 \mathrm{~g} / \mathrm{mol}$, and purity of $95 \%$ which was supplied by IRO Group Inc., China. The non-ionic high molecular weight Polyethylene oxide powder PEO $8 \times 106 \mathrm{~g} / \mathrm{mol}$ was supplied by CHINA DEXIN CO., LTD, China. In addition, the deionised (DI) water of (electrical conductivity of $1-3 \mathrm{mS} / \mathrm{cm}$ ) is utilized as solvents for polymer, and polymersurfactant mixtures. The concentration range of PEO stock solutions was prepared 0.001 to $0.003 \mathrm{wt} \%$ in $4500 \mathrm{ml}$ deionised water. A magnetic stirrer plate was utilized for mixing the polymer in distilled water at enough low speed for avoiding break down of polymer molecules and remains at stirrer for 24 hours to ensure the polymer is completely distributed in solution. An appropriate amount of 0.1 to $0.5 \mathrm{wt} \%$ stock solution of SDBS was added to the polymer solution to get polymer-surfactant complex.

\subsection{Rotating Disk Apparatus Experiment}

Rotating disc methods are a simpler alternate to pulling tanks and flow tests for depicting the drag properties of test batches [15]. The rotating disk apparatus RDA in the present work is designed and fabricated in order to testing the drag reduction efficiency of the investigated liquids by applying shearing force and measuring the torque. Discs are easily rotated in a tank of water, at high velocities. The RDA design is shown in Figure 1 with full details.

To achieve the degradation performance at the rotating disk apparatus, by measuring the torque (mN.M) of disk rotate at a certain speed $(1 / \mathrm{min})$ for the pure solvent (distilled water) and the solution in different concentration. The estimated of drag reduction percentage (\% DR) by the equation as follows:

$$
\% \mathrm{DR}=\left(\frac{\mathrm{T}_{\mathrm{D}}-\mathrm{T}_{\mathrm{S}}}{\mathrm{T}_{\mathrm{D}}}\right) * 100
$$

where, $\left(T_{D}\right)$ is the torque in the distilled water either $(T S)$ is the torque in the dilute solution. The torque unit by (mN.M) at a speed of $1800 \mathrm{rpm}$ is measured in RDA system the flow reach to turbulent state when the speed of rotation disk $(r p m>650)$. Moreover, all tests have been carried out at constant temperature $25^{\circ} \mathrm{C}$. [16]. 


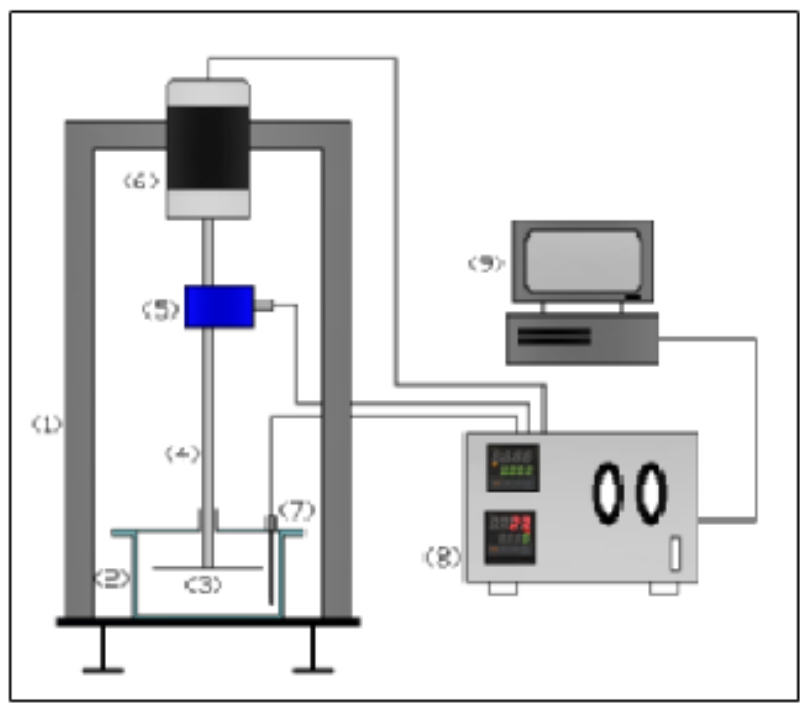

Fig. 1. Schematic diagram of the rotating disk apparatus for drag reduction measurement: (1) Outside frame, (2) Fluid container, (3) Rotating disk, (4) Disk holding shift, (5) Torque sensor, (6) Electric motor, (7) thermocouple, (8) Controller interface, and (9) PC..

\section{Result and Discussion}

The mechanical degradation of PEO was investigated in this paper using a RDA. The RDA measures changes in DR as a function of time and the concentration of SDBS surfactant in a turbulent flow. Figure 2 depicts the DR as a function of time for four different SDBS concentrations $(100,200,400$, and $500 \mathrm{ppm})$ with $10 \mathrm{ppm}$ of PEO at a rotational speed of $1800 \mathrm{rpm}$ and indicates that the DR decreases with time due to the degradation of the polymer molecules under turbulent flow. The time-dependence drag reduction of PEO at different concentrations is plotted in figure $2 \mathrm{a}$. in 3D form, taking the time zero for maximum drag reduction. This figure indicates clearly that low concentrations will be degraded quickly compared with high concentration i.e $500 \mathrm{ppm}$. Therefore, the percentage of DR decreases rapidly and approaching to zero value after 600 seconds for $100 \mathrm{ppm} 300 \mathrm{ppm}$. While at $500 \mathrm{ppm}$ concentration there is still having better resistance.
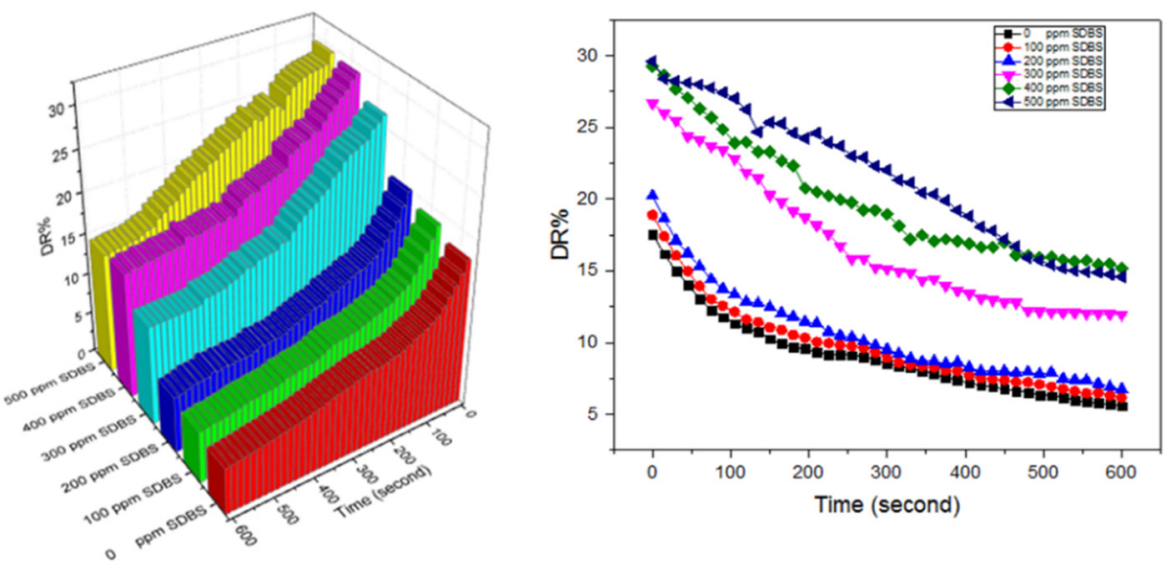

Fig. 2. The degradation measurement obtained from the experiment data by using the drag reduction equation with time for $10 \mathrm{ppm}$ PEO. 
Further investigation on the degradation improvement of the polymer was conducted at concentration of $20 \mathrm{ppm}$ of PEO. Figure 3 shows the result obtained for the $20 \mathrm{ppm}$ PEO at five different concentrations of SDBS surfactant as function of drag reduction. It was observed that with the addition of surfactant to the PEO the degradation resistance of the PEO was improved. The result also shows higher drag reduction compare to that obtained at $10 \mathrm{ppm}$. For instant, at $10 \mathrm{ppm}$ PEO and $500 \mathrm{ppm}$ SDBS the drag reduction value reduced by $50 \%$ from the initial value. On the other hand, at 20 ppm PEO and 500 SDBS the drag reduction has been decreased by $30 \%$ only. This is due to the increase in the number of aggregates form during the interaction between polymer and surfactant. This also can be attributed to increase the polymer concentration in solution.
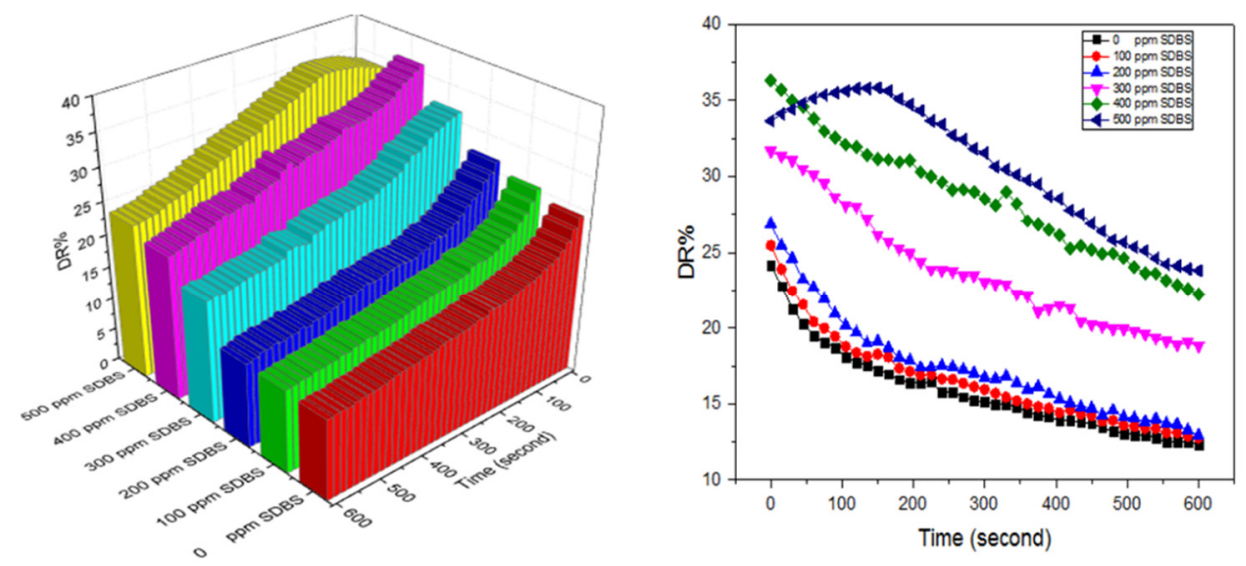

Fig. 3. The degradation measurement obtained from the experiment data by using the drag reduction equation with time for $20 \mathrm{ppm}$ PEO.

At $40 \mathrm{ppm}$ of PEO the degradation resistant was further improved as illustrated in figure 4. The change in degradation resistance behaviour was observed starting at low concentrations such as $100 \mathrm{ppm}$ SDBS. Further improvement was obtained with addition of $200 \mathrm{ppm}$ and $300 \mathrm{ppm}$ of SDBS to $40 \mathrm{ppm}$ of PEO. This result was higher than that obtained at $20 \mathrm{ppm}$. However, at 400 and $500 \mathrm{ppm}$ SDBS again the result was in contrary to expected behaviour, the degradation resistance increased initially till reached to saturation point, and then start to degrade following the same trend as the low concentrations. This also can be attributed to the shift of the total viscosity of the solution which in fact effects the drag reduction as well improve the degradation resistant 

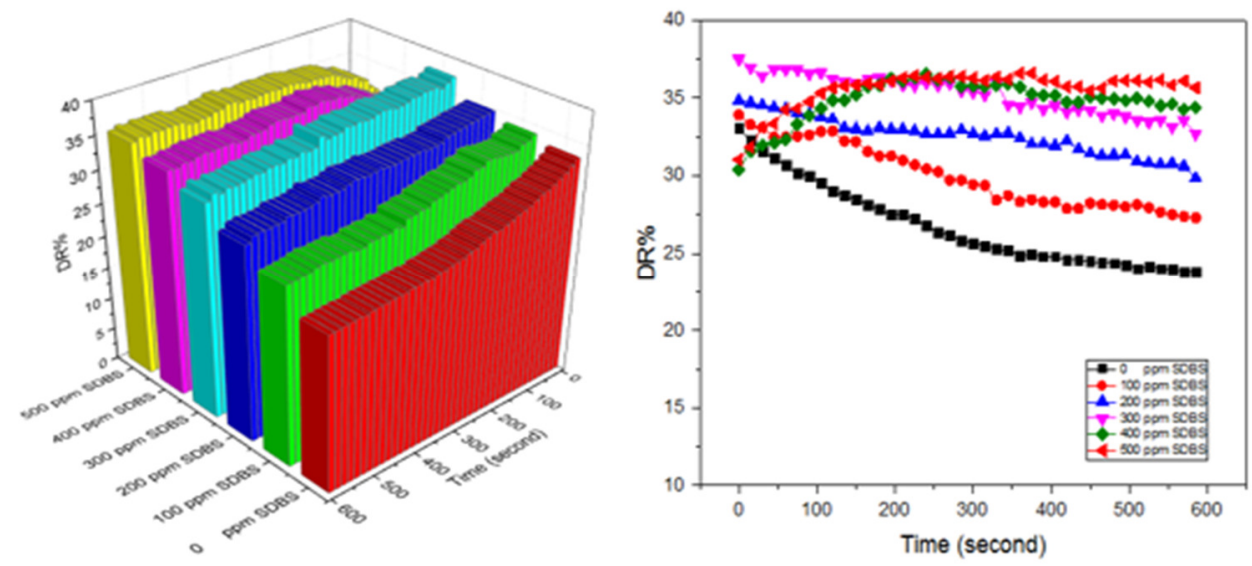

Fig. 4. The degradation measurement obtained from the experiment data by using the drag reduction equation with time for $40 \mathrm{ppm}$ PEO.

At $60 \mathrm{ppm}$ of PEO the interaction between the polymer and surfactant was observed to have different behaviour in term of degradation resistance. Figure 5 shows drag reduction the result obtained at $60 \mathrm{ppm}$ of PEO and 100, 200, 400 and $500 \mathrm{ppm}$ of SDBS as function of time. It was observed that at low concentrations of SDBS the degradation resistance improvement still better than that at $40 \mathrm{ppm}$ of PEO. However, at higher concentrations of PEO the resulting improvement decreased at higher SDBS concentration i.e. 400 and 500 $\mathrm{ppm}$. This due to the effect of internal repulsion extruded by the increase of the attached surfactant into the polymer solution which results in contrary effects.
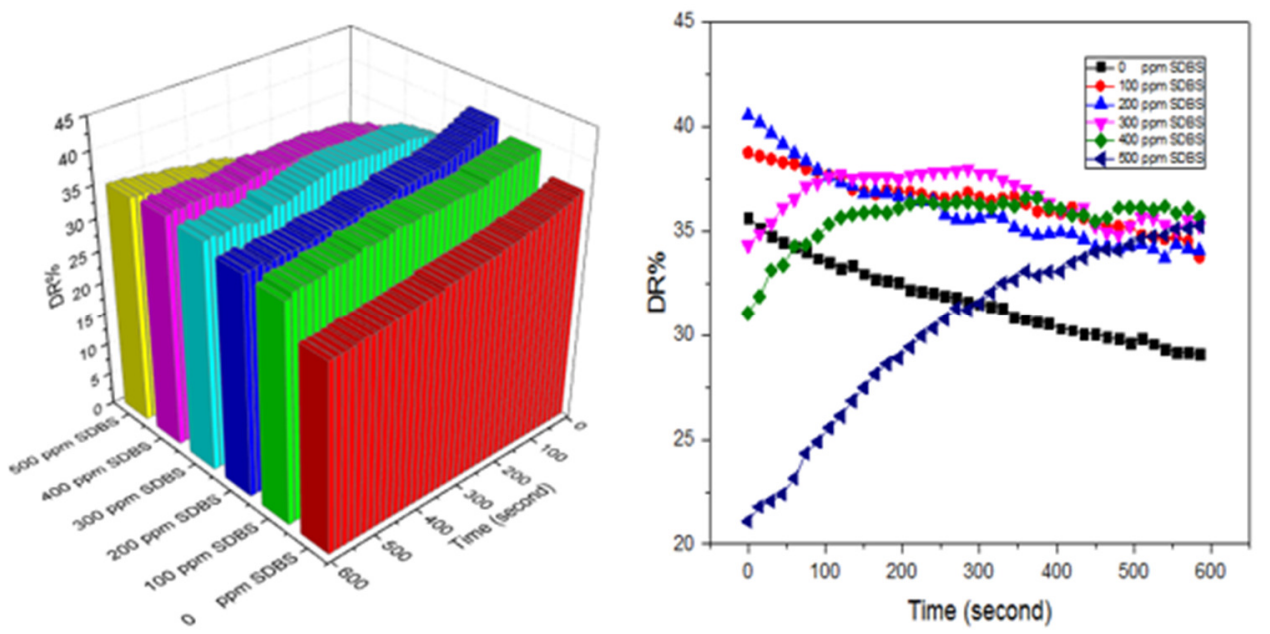

Fig. 5. The degradation measurement obtained from the experiment data by using the drag reduction equation with time for $60 \mathrm{ppm}$ PEO.

\section{CONCLUSION}

In this research work, new aspects of polymer-surfactant complex have been studied experimentally, in the presence of surfactants into polymer solution under extreme shear rate by using rotating disk apparatus. The degradation ability of the polymer was observed to 
increase with the addition of the surfactant compare to the polymer alone. Further, this improvement was noticed to increase with the increase in the surfactant concentration at low PEO concentration i.e. 10 and $20 \mathrm{ppm}$. At higher concentrations of PEO the resulting improvement decreased at higher SDBS concentration i.e. 400 and $500 \mathrm{ppm}$. This due to the effect of internal repulsion extruded by the increase of the attached surfactant into the polymer solution which result in contrary effects.

This work was supported by University Malaysia Pahang (UMP) equipment and Ministry of higher education and scientific research in Iraq through the scholarship.

\section{References}

1. B.A. Toms, in: Proceedings of the 1st International Congress on Rheology, 2, 135141 (1949).

2. H.A. Abdulbari, A. Shabirin, and H.N. Abdurrahman, J. Ind. Eng. Chem., 20(4), 1157-1170. (2014).

3. M. Escudier, F. Presti, and S. Smith, J. Non-Newton Fluid, 81(3), 197-213 (1999).

4. M.T. Islam, S.A. Vanapalli, and M.J. Solomon, Macromolecules, 37(3), 1023-1030 (2004).

5. I.M. Jánosi, D. Jan, K.G. Szabó, T. Tél, Exp. Fluids, 37(2), 219-229 (2004).

6. Sellin, R., J. Hoyt, O. Scrivener, J. Hydraul Res., 20(1), 29-68 (1982).

7. H.J. Choi, C.A. Kim, J.I. Sohn, M.S. Jhon, Polym. Degrad. Stab., 69(3), 341-346 (2000).

8. G.A. Khan, M. Shaheruzzaman, M.H. Rahman, S.M. Abdur Razzaque, M. Sakinul Islam,M. Shamsul Alam. Fiber Polym., 2009. 10(1), 65-70.

9. D.A. da Silva, R.C. de Paula, J.P. Feitosa, Eur. Polym. J., 43(6), 2620-2629 (2007).

10. H.A. Abdulbari, E. Faraj, J. Gimbun, W.K. Mahmood. Adv. Appl. Fluid Mech., 18(1), 113 (2015).

11. E. Akindoyo, H. Abdulbari, J. Appl Fluid Mech., 9(3), 1041-1049 (2016).

12. Z. Matras, T. Malcher, B. Gzyl-Malcher, Thin Solid Films, 516(24), 8848-8851 (2008).

13. J.T. Kim, C.A. Kim, K. Zhang, C.H. Jang, H.J. Choi, Colloid Surface A., 391(1), 125-129 (2011).

14. A.A. Mohsenipour, and R. Pal, Ind. Eng. Chem. Res., 52(3), 1291-1302 (2013).

15 E. Holm, M. Schultz, E. Haslbeck, W. Talbott. Biofouling, 20(4-5), 219-226 (2004).

16. H.J. Choi, M.S. Jhon,. Ind. Eng. Chem. Res., 35(9) 2993-2998 (1996). 